\title{
EXTREME POINTS OF VECTOR FUNCTIONS
}

\section{SAMUEL KARLIN}

Several previous investigations have appeared in the literature which discuss the nature of the set $T$ in $n$-dimensional space spanned by the vectors $\left(\int_{S} d \mu_{1}, \cdots, \int_{S} d \mu_{n}\right)$ where $S$ ranges over all measurable sets. It was first shown by Liapounov that if $\mu_{1}, \cdots, \mu_{n}$ are atomless measures, then the set $T$ is convex and closed. Extensions of this result were achieved by D. Blackwell [1] and Dvoretsky, Wald, and Wolfowitz [2]. A study of the extreme points in certain function spaces is made from which the theorems concerning the range of finite vector measures are deduced as special cases. However, the extreme point theorems developed here have independent interest. In addition, some results dealing with infinite direct products of function spaces are presented. Many of these ideas have statistical interpretation and applications in terms of replacing randomized tests by nonrandomized tests.

1. Preliminaries. Let $\mu$ denote a finite measure defined on a Borel field of sets $\mathfrak{F}$ given on an abstract set $X$. Let $L(\mu, \mathfrak{F})$ denote the space of all integrable functions with respect to $\mu$. Finally, let $M(\mu, \mathfrak{F})$ be the space of all essentially bounded measurable functions defined on $X$. It is well known that $M(\mu, \mathfrak{F})$ constitutes the conjugate space of $L(\mu, \mathfrak{F})$ and consequently the unit sphere of $M(\mu, \mathfrak{F})$ is bicompact in the weak ${ }^{*}$ topology [3]. Let $\left(\otimes L^{n}\right)$ denote the direct product of $L$ taken with itself $n$ times and take $\left(\otimes M^{n}\right)$ as the direct product of $M n$ times. With appropriate choice of norm, $\left(\otimes M^{n}\right)$ becomes the conjugate space to $\left(\otimes L^{n}\right)$. Another description of $\left(\otimes M^{n}\right)$ is that it consists of all $n$-vectors, each component of which is an essentially bounded measurable function. In notation, let $x \in\left(\otimes M^{n}\right)$ be denoted by $\bar{x}=\left(x_{i}(t)\right), i=1, \cdots, n$, with $x_{i}$ in $M$.

2. Extreme points in $M_{A}$. Let $A$ be a bounded closed convex set in Euclidean $n$-space. Let $M_{A}$ consist of all $x$ in $\left(\otimes M^{n}\right)$ whose range of values lie almost everywhere in $A$. I.e., for almost every $t, \bar{x}(t)=\left(x_{i}(t)\right)$ is in $A$. Let $B$ consist of the extreme points of $A$, and let $\bar{B}=$ closure of $B$. For $n \geqq 3$, it is not necessarily true that $\bar{B}=B$.

In view of the convexity of $A$, the set $M_{A}$ is easily verified to be convex, bounded, and weak * closed. We indicate the proof of this last fact. Let $\bar{x}_{0}$ represent a weak ${ }^{*}$ limit point of $M_{A}$. Suppose $\bar{x}_{0} \notin M_{A}$. This implies the existence of a set of positive finite measure

Received by the editors October 11, 1952 and, in revised form, October 30, 1952. 
$E$ with $\bar{x}_{0}(E) \notin A$. Since a denumerable number of hyperplanes determine $A$, it is easy to construct a plane $\left\{\xi_{i}\right\}$ so that $\sum_{i=1}^{n} \xi_{i} \eta_{i}>c$ for $\left\{\eta_{i}\right\} \in A$ while $\sum_{i=1}^{n} \xi_{i} x_{i}^{0}\left(E_{1}\right)<c-\epsilon$ for a set of positive measure $E_{1} \subset E$. Let $\bar{w}(t)$ denote the function in $\otimes L^{n}(\mu, \mathfrak{F})$ defined as follows:

$$
\bar{w}(t)=\left(w_{i}(t)=\left\{\begin{array}{cc}
\xi_{i} / \mu\left(E_{1}\right) & \text { for } t \text { in } E_{1} \\
0 & \text { for } t \notin E_{1}
\end{array}\right\}\right) .
$$

We note that $(\bar{w} \cdot \bar{x})=\sum_{i=1}^{n} \int w_{i} x_{i} d \mu$ represents the inner product and is $\geqq c$ for $\bar{x}$ in $M_{A}$, while $\left(\bar{w} \cdot \bar{x}_{0}\right)<c-\epsilon$. This shows that $\bar{x}_{0}$ cannot be a weak ${ }^{*}$ limit point of $M_{A}$, a contradiction from which we conclude that $\bar{x}_{0} \in M_{A}$.

Since the unit sphere $\left(\otimes M^{m}\right)$ is bicompact in the weak * topology, we deduce that $M_{A}$ is bicompact. On account of the bicompactness and convexity, the Krein-Milman theorem guarantees the existence of extreme points in $M_{A}$. The first theorem characterizes such extreme points. We define $M_{\bar{B}}$ in a similar manner to $M_{A}$.

THEOREM 1. The extreme points of $M_{A}$ are contained in $M_{\bar{B}}$.

Proof. Let $\bar{x}_{0}=\left(x_{i}^{0}\right)$ be an extreme point of $M_{A}$ and let us suppose the contrary that $\bar{x}_{0}$ is not in $M_{\bar{B}}$. There exists an $\epsilon_{0}$ such that $\bar{x}_{0} \notin M_{\bar{B}}\left(\epsilon_{0}\right)$ where $\bar{B}\left(\epsilon_{0}\right)$ consists of the closure of the set of points of $A$ obtained from $\bar{B}$ by describing an $\epsilon_{0}$ sphere about every point in $B$. In fact, otherwise, let $\epsilon_{n} \rightarrow 0$ and suppose $\bar{x}_{0} \in M_{\bar{B}_{\left(\epsilon_{n}\right)}}$ for every $n$. That is, except for a set $E_{n}$ of $\mu$ measure zero the range of values of $\bar{x}_{0}$ is in $M_{\bar{B}_{\left(\epsilon_{n}\right)}}$. Since $\mu\left(\sum E_{n}\right)=0$, we deduce that $\bar{x}_{0}$ is in $M_{\cap \bar{B}\left(\epsilon_{n}\right)}=M_{\bar{B}}$, which serves as a contradiction. Thus there exists a set $E$ of positive measure such that $\bar{x}_{0}(t)$, for $t$ in $E$, is not in $\bar{B}\left(\epsilon_{0}\right)$. It follows easily that a constant vector $\bar{a}$ exists of small magnitude such that $\bar{x}_{0} \pm \bar{a}$ is in $A$ when $t$ is in $E_{0} \subset E$. Put

$$
\boldsymbol{\phi}(t)= \begin{cases}\bar{a}, & t \in E_{0}, \\ 0, & t \notin E_{0} .\end{cases}
$$

Consequently, $\bar{x}_{0}=\left(\bar{x}_{0}+\bar{\phi}\right) / 2+\left(x_{0}-\bar{\phi}\right) / 2$ with $\bar{x}_{0}+\bar{\phi}, \bar{x}_{0}-\bar{\phi}$ in $M_{A}$. This impossibility establishes the result, Q.E.D.

REMARK. The set $M_{\bar{B}}$ need not be contained in $\left(M_{A}\right)^{(e)}$ (extreme points) but $M_{B}$ is.

3. Extreme points of $M_{A}$ with side conditions. A finite measure $\mu$ is said to be atomless if for any measurable set $E$ of positive measure $\mu$ there exists a measurable subset $E_{0}$ such that $0<\mu\left(E_{0}\right)<\mu(E)$. The theorem of Liapounov [4] states that if $\mu_{1}, \cdots, \mu_{n}$ are atom- 
less finite measures defined on the same Borel field of sets, then the span in Euclidean $n$ space of the $n$ tuples $\left(\int x(t) d \mu_{j}(t)\right)$ obtained by allowing $x(t)$ to range over all characteristic functions is convex and closed. Therefore, it follows that the set of all $n$ tuples $\left(\int x(t) d \mu_{j}(t)\right)$ with $0 \leqq x(t) \leqq 1$ spans the same set. An apparent weaker formulation of the above spanning result which is, however, equivalent is that for any given measurable set $S$ there exists a set $E \subset S$ such that simultaneously $\mu_{i}(E)=\mu_{i}(S) / 2$ for $i=1, \cdots, n$.

Let $M\left[A, \mu_{j}\right]=\left[\bar{\xi}_{i}, i=1, \cdots, n\right]$ with $\xi_{i}=\int \overline{\mathbf{x}}(t) d \mu_{i}(t)$ and $\overline{\mathbf{x}}$ in $M_{A}$. It is of course understood that $x$ is measurable with respect to all $\mu_{j}$. This set can also be viewed as points in Euclidean $n m$ space.

THEOREM 2. If $\mu_{i}(i=1, \cdots, n)$ consist of atomless finite measures, then the extreme points of the set $T$ of all $\bar{x}(t)$ measurable $\mu_{i}(i=1, \cdots, n)$ satisfying $\bar{x} \in M_{A}$ and $\bar{b}_{i} \leqq \int \bar{x} d \mu_{i} \leqq \bar{a}_{i}$ are contained in $M_{\bar{B}}$.

REMARK 1. The vector inequality $\int \bar{x} d \mu_{i} \leqq \bar{a}_{i}$ means that the inequality holds for each component of the vectors.

REMARK 2. The interest of Theorem 2 is that if we impose linear side conditions on the set $M_{A}$ of the form $\bar{b}_{i} \leqq \int \bar{x} d \mu_{i} \leqq \bar{a}_{i}$, then no new extreme points are added to this convex subset of $M_{A}$ when $B$ $=\bar{B}$. Only the set of extreme points of $M_{A}$ may be diminished. This result is in sharp contrast to the situation in the case of atomic measures. We leave it to the reader to construct examples involving atomic measures which violate the conclusions of the theorem.

REMARK 3. If $\mu=\mu_{1}+\cdots+\mu_{n}$, then by virtue of the RadonNikodym theorem $\mu_{i}(E)=\int_{E} f_{i}(t) d \mu(t)$. The space $M(\mu, \mathfrak{F})$ considered here is the set of all bounded measurable functions $x(t)$, which is the conjugate space to $L(\mu, \mathfrak{F})$ where $\mathfrak{F}$ is the common Borel field of sets over which all the $\mu_{i}$ are defined. Therefore, bounded weak ${ }^{*}$ closed sets in $M(\mu, \mathfrak{F})$ are bicompact and the same holds for $\left(\otimes M(\mu, \mathfrak{F})^{n}\right)$. The statement of the theorem in terms of the $f_{i}$ is that the set $\Gamma$ of extreme points of all $\bar{x}(t)$ in $M_{A}$ which satisfy $\bar{b}_{i} \leqq \int \bar{x} f_{i}(t) d \mu(t) \leqq \bar{a}_{i}$ is contained in $M_{\bar{B}}$ when $\mu$ is atomless. Since $A$ is convex and closed and the linear restrictions are generated by elements of $L(\mu, \mathfrak{F})$, we deduce that $T$ is bicompact and convex and hence possesses extreme points. The theorem thus determines the form of such extreme points.

Proof of Theorem 2. Let $\bar{x}_{0}$ be an extreme point of $T$ and suppose $\bar{x}_{0}$ is not in $M_{\bar{B}}$. Then an argument as in Theorem 1 shows that for some positive $\epsilon_{0}$ the point $\bar{x}_{0}$ is not in $M_{\bar{B}_{\left(\epsilon_{0}\right)}}$. Consequently there exists a set $S$ of positive measure $\mu$ for which $x_{0}(t) \notin \bar{B}\left(\epsilon_{0}\right)$ for $t$ in $S$. As in Theorem 1 , one can find a constant vector $\bar{c}$ and a measurable set $E C S$ of positive $\mu$ measure such that $\bar{x}_{0} \pm \bar{c}$, for $t$ in $E$, is contained 
in $A$. An application of the theorem of Liapounov yields a measurable subset $E_{0}$ of $E$ with

$$
\mu_{i}\left(E_{0}\right)=\int_{E_{0}} f_{i} d \mu=\frac{1}{2} \int_{E} f_{i} d \mu=\frac{1}{2} \mu_{i}(E) \quad \text { for } i=1, \cdots, n .
$$

Put

$$
\Phi(t)=\left\{\begin{aligned}
\bar{c}, & t \text { in } E_{0}, \\
-\bar{c}, & t \text { in } E-E_{0}, \\
0 & \text { elsewhere, }
\end{aligned} \text { and } \quad \bar{\psi}(t)=\left\{\begin{aligned}
-\bar{c}, & t \text { in } E_{0}, \\
\bar{c}, & t \text { in } E-E_{0} \\
0 & \text { elsewhere }
\end{aligned}\right.\right.
$$

It is easy to verify that $\bar{x}_{0}=\left(\bar{x}_{0}+\phi\right) / 2+\left(\bar{x}_{0}+\psi\right) / 2$ where $\bar{x}_{0}+\bar{\phi}$ and $\bar{x}_{0}+\bar{\psi}$ are in $M_{A}$. A simple computation gives that

$$
\begin{aligned}
\int\left(\bar{x}_{0}+\bar{\phi}\right) f_{i} d \mu & =\int \bar{x}_{0} f_{i} d \mu+\bar{c}\left[\int_{E_{0}} f_{i} d \mu-\int_{E-E_{0}} f_{i} d \mu\right] \\
& =\int \bar{x}_{0} f_{i} d \mu+\bar{c}\left[\frac{1}{2} \int_{E} f_{i} d \mu-\frac{1}{2} \int_{E} f_{i} d \mu\right] \\
& =\int \bar{x}_{0} f_{i} d \mu .
\end{aligned}
$$

Similarly, $\int\left(\bar{x}_{0}+\psi\right) f_{i} d \mu=\int \bar{x}_{0} f_{i} d \mu$. Therefore $\bar{x}_{0}+\psi$ and $\bar{x}_{0}+\Phi$ satisfy the linear inequalities and hence lie in $T$. We have exhibited a contradiction of the extreme point nature of $\bar{x}_{0}$ and the proof of the theorem is hereby complete.

Although we employed the convexity part of the theorem of Liapounov, we can now use Theorem 2 to obtain an extension of the theorem of Liapounov. It is to be remarked, however, that the proof of convexity in the Theorem of Liapounov is the simpler result to obtain and is also used in [1] and [2].

Theorem 3. Let $\mu_{j}(j=1, \cdots, n)$ be atomless, finite measures. Then $M\left[A, \mu_{j}\right]=M\left[\bar{B}, \mu_{j}\right]$.

REMARK 4. An immediate conclusion of this theorem is that the range in $E^{n m}$ of $\int \bar{x}(t) d \mu_{j}(t)$ where $\bar{x}(t)$ ranges over $M_{\bar{B}}$ is convex and closed. This follows from the evident convexity and closedness of $M\left[A, \mu_{j}\right]$ (see Remark 3).

Proof. Let $\bar{x}_{0}$ be any point in $M_{A}$. Put $\bar{\xi}_{i}=\int \bar{x}_{0} d \mu_{i}$. Let $\Gamma$ be the set of all $\bar{x}$ in $M_{A}$ for which $\bar{\xi}_{i}=\int x d \mu_{i}$. The set is weak * closed and convex and hence bicompact (see Remark 3 ). There exists consequently an extreme point $\bar{x}_{1}$ of $\Gamma$. By virtue of Theorem 2, $\bar{x}_{1}$ is in 
$M_{B}$ and $\int \bar{x}_{1} d \mu_{i}=\xi_{i}=\int \tilde{x}_{0} d \mu_{i}$. Thus, we have shown that any point in $M\left[A, \mu_{j}\right]$ is in $M\left[\bar{B}, \mu_{j}\right]$. On the other hand since $\bar{B} \subset A$, evidently we get $M\left[\bar{B}, \mu_{j}\right] \subset M\left[A, \mu_{j}\right]$. Combining we have established our result.

COROLLARY 1 [1]. Let $C$ represent any closed bounded set in $E^{m}$, then $M\left[C, \mu_{i}\right]$ is convex and closed if the $\mu_{j}$ are atomless.

Proof. Let $A$ be the convex span of $C$. Clearly $A$ is closed and $\bar{B} \subset C$. Evidently, $M\left[\bar{B}, \mu_{i}\right] \subset M\left[C, \mu_{i}\right] \subset M\left[A, \mu_{i}\right]$. However, Theorem 3 implies the equality of the outside two sets which thus gives the result (sea Remark 4).

As was pointed out in [1] and [2] the closure of $M\left[C, \mu_{i}\right]$ can be established under any circumstances regardless of the nature of the measures $\mu_{i}$. In fact, any measure $\mu$ can be expressed as a sum of an atomless measure $\mu^{*}$ and a countable union of pure atomic measures which we designate by $\bar{\mu}$. In notation, $\mu=\mu^{*}+\bar{\mu}$. In the case of $\bar{\mu}$ one can show directly the closure $[1 ; 2]$. The case of $\mu^{*}$ was handled in Theorem 3. Thus, to obtain the closure property for the general case one needs only to apply this decomposition result to the measure $\mu=\mu_{1}+\mu_{2}+\cdots+\mu_{n}$, and invoke the above remarks to the parts of $\mu$. Precisely: $M\left[C, \mu^{*}+\bar{\mu}\right]=$ convex span $\left(M\left[c, \mu^{*}\right], M[c, \bar{\mu}]\right)$.

Closure properties of extreme points. This section investigates whether the set of extreme points of $M_{A}$ is weak * closed or weakly closed. The measure $\mu$ is taken to be atomless and $A$ is assumed to contain more than one point.

THEOREM 4. Let $B=\bar{B}$, then the set of extreme points of $M_{A}$ is not weak ${ }^{*}$ closed and the extreme points $M_{B}$ and $M_{A}$ are sequentially weakly closed.

Proof. We suppose that the set of extreme points of $M_{A}$ is weak ${ }^{*}$ closed. Let $\bar{x}$ denote any element of $M_{\mathbf{A}}$ and consider any integrable $f_{1}(t), \cdots, f_{n}(t)$. Theorem 2 provides an extreme point $\bar{x}_{0}$ such that $\int f_{i} \bar{x} d \mu=\int f_{i} \bar{x}_{0} d \mu$ for $i=1, \cdots, n$. Of course, $\bar{x}_{0}$ depends on the choice of $f_{1}, \cdots, f_{n}$. Put $G\left(f_{\alpha}\right)=\left[\bar{x}_{0} \mid \bar{x}_{0}\right.$ an extreme point of $M_{A}$ and $\left.\int \bar{x}_{0} f_{\alpha} d \mu=\int \tilde{x} f_{\alpha} d \mu\right]$. The assumption implies that $G\left(f_{\alpha}\right)$ is weak ${ }^{*}$ closed and nonempty. The above argument shows that every finite intersection of $G\left(f_{\alpha_{i}}\right)$ is weak * closed and nonempty. Since the extreme points of $M_{A}$ are weak ${ }^{*}$ closed and hence bicompact, we have $L=\bigcap G\left(f_{\alpha}\right) \neq 0$. Let $\bar{x}_{0}$ be in $L$, then $\int \bar{x} f d \mu=\int \bar{x}_{0} f d \mu$ for every integrable $f$ and hence $\bar{x}=\bar{x}_{0}$ almost everywhere. Hence every $\bar{x}$ in $M_{A}$ is a point in $M_{\bar{B}}$, which is clearly impossible since $A$ consists of more than one point. We now verify the second assertion of the theorem. If $\bar{x}_{n}$ con- 
verges weakly to $\bar{x}$, then at least $\bar{x}_{n}$ converges almost everywhere to $\bar{x}$ and $\left\|\bar{x}_{n}\right\|<C$. Thus if $\bar{x}_{n}$ are in $M_{B}$, then clearly $\bar{x}$ is in $M_{B}$ as $B$ is closed. This completes the proof of the theorem.

REMARK 5. The same result concerning the extreme points can be carried over to sets studied in Theorem 2. Furthermore, in the case that $\mu$ is purely atomic and $B=\bar{B}$, it is easily seen that the set of extreme points $M_{B}$ of $M_{A}$ is weak ${ }^{*}$ closed. We omit the details. This is in contrast with Theorem 4.

A further example more clearly illustrating the conclusion of Theorem 4 is now given explicitly. On the basis of Theorem 1, it follows that the extreme points of the set of all Lebesgue measurable positive functions bounded by one consist of all characteristic functions. In particular, $s_{n}(t)=1 / 2+r_{n}(t) / 2$, where $r_{n}(t)=\operatorname{sign} \sin 2^{n+1} \pi t$, is an extreme point for each $n$, where $r_{n}(t)$ are the classical Rademacher functions. The orthogonality and boundedness of $r_{n}(t)$ imply for any integrable function $f(t)$ that $\lim _{n \rightarrow \infty} \int_{0}^{1} f(t) r_{n}(t) d t=0$. Consequently $s_{n}(t)$ converges weak $*$ to the function identically equal to $1 / 2$ which is clearly not an extreme point.

Infinite vector functions. The space $M^{\infty}=\left(\otimes M(\mu, \mathfrak{F})^{\infty}\right)$ is defined as the countable infinite direct product of $M(\mu, \mathfrak{F})$. An element of $M^{\infty}$ has the form $\bar{x}=\left(x_{1}(t), x_{2}(t), \cdots\right)$ where each $x_{i}(t)$ is in $M(\mu, \mathfrak{F})$. Let $(m)$ denote the Banach space consisting of all bounded sequences. It is well known that $(m)$ is the conjugate space of $(l)$ (sequences which converge absolutely). Therefore bounded weak ${ }^{*}$ closed sets in $(m)$ are bicompact and convex bounded weak * closed sets are spanned in the weak * topology by the extreme points. This is a restatement of the Krein-Milman Theorem.

We discuss an example to indicate the extensions of the preceding theory to the infinite case.

A. Let $\bar{x}=\left(x_{i}(t)\right), i=1,2, \cdots$, with $0 \leqq x_{i}(t) \leqq 1$. Let $M_{A}$ denote the set of all such points in $M^{\infty}$. It can be shown in a manner similar to the proof of Theorem 1 that the extreme points of $M_{A}$ consist of those elements $\bar{x}$ whose value for almost every $t$ lie in the extreme points of the set $B$ in $(m)$ which consists of sequences $\left(m_{i}\right)$ with $m_{i}=0$ or 1 . Also $M_{A}$ is weak * closed. Furthermore, it follows that if additional linear conditions $\int \bar{x} d \mu_{j}=\bar{\alpha}_{j}, j=1, \cdots, m$, are imposed with $\mu_{j}$ atomless, then no new extreme points are added. This yields as in Theorem 3 the convexity and weak * closure of the span in $(m)$ of $\left(\int \bar{x} d \mu_{j}\right)$.

More detailed results on the infinite vector functions will appear in a later publication. 
Extreme points in measure spaces. It is of in terest to compare the type of results obtained above with the description of the set of extreme points in other Banach spaces where constraints are present. Specifically, we study the extreme points of the set of all positive regular measures of total variation one defined on a bi-compact Hausdorff space $X$ subject to linear constraints.

Let $h_{1}(x), \cdots, h_{n}(x)$ be continuous functions defined on $X$. We look to characterize the set of extreme points of the set $L$ of all positive measures $=$ distributions $\mu$ with $\int d \mu=1, \int h_{i}(x) d \mu=a_{i}$. If we consider the image $M$ in Euclidean $n$-space of $\mu \rightarrow\left\{\int h_{i}(x) d \mu\right\}$, then it follows easily that $M$ consists of the set of all points, in the convex span of the bi-compact image $\Gamma$ of $X$ given in parametric form by $t_{i}=h_{i}(x), i=1, \cdots, n$, with $x$ in $X$. Indeed, let $C$ represent the convex span of $\Gamma$. As the convex span of the compact set $\Gamma, C$ is closed. If $\bar{t}$ denotes a point in $M$ but outside $C$, then there exists a hyperplane which separates $C$ and $\bar{t}$. Therefore constants $b_{i}$ exist so that $\sum b_{i} \bar{t}_{i}<-\delta<0$ and $\sum b_{i} h_{i}(x) \geqq 0$ for each $x$ in $X$. Since $\bar{t}_{i}$ $=\int h_{i}(x) d \mu(x)$ for some $\mu$, then $0>\sum b_{i} \bar{t}_{i}=\int \sum b_{i} h_{i}(x) d \mu(x) \geqq 0$, which is impossible. This shows that $C=M$.

We now specialize the functions $h_{i}(x)$ so that for any choice of $n$ points $x_{1}, x_{2}, \cdots, x_{n}$ the rank of the matrix $h_{i}\left(x_{j}\right)$ is $n$. Under this condition we now show that points on the boundary of $M$ come from unique measures possessing only a finite number of points of increase. To this end, let $\bar{t}$ be a point on the boundary of $M$ whose components are given by $t_{i}=\int h_{i}(x) d \mu_{0}(x)$. There exists a supporting plane to $M$ at $\bar{t}$. Hence, $\int \sum b_{i} h_{i}(x) d \mu_{0}(x)=0$ for appropriate constants and, since $\sum b_{i} h_{i}(x) \geqq 0$, we get for every point $x$ of increase of $\mu_{0}$ that $\sum b_{i} h_{i}(x)$ $=0$. This implies in view of the property of $h_{i}(x)$ that at most $n$ points of increase are possessed by $\mu_{0}$. Since $\sum_{r=1}^{n} \lambda_{i} h_{j}\left(x_{i}\right)=t_{j}$ where $h_{i}\left(x_{j}\right)$ has rank $n$ we obtain that the $\lambda_{i}$ are unique. This establishes the assertion that boundary points of $M$ correspond to unique measures.

Finally, we investigate the form of the set of extreme points of $L$. It is clear that the linear constraints $\int h_{i}(x) d \mu(x)=a_{i}$ define geometrically a section $K$ of the convex set $M$ and that extreme points of $K$ must be boundary points of $M$. Thus any extreme point of $K$ is built up of a convex finite sum of pure atomic measures. Without loss of generality, we can therefore restrict our considerations only to measures $\mu$ which are finite convex combinations of pure atomic measures. Let $h_{0}(x) \equiv 1$. We now suppose, in addition to the conditions stated before, that the rank of the determinant $h_{i}\left(x_{j}\right)$ for $n+1$ distinct points $x_{j}$ is $n+1, i=0,1, \cdots, n$. Suppose now that $\mu$ 
$=\sum \alpha_{i} \mu_{i}$ with $\mu_{i}$ in $L\left(\alpha_{i} \geqq 0\right)$. Clearly any point of increase for a single $\mu_{i}$ is shared by $\mu$. Let $x_{1}, \cdots, x_{m}$ denote the totality of points of increase of $\mu$. Since $\int h_{i}(x) d \mu(x)=a_{i}$ for $i=1, \cdots, n$ and $\int h_{0}(x) d \mu(x)$ $=1$, we obtain that

$$
\sum_{i=1}^{m} \lambda_{i} h_{j}\left(x_{i}\right)=a_{j}, \quad i=0,1, \cdots, n,
$$

where $a_{0}=1$. Since the rank of $\left[h_{j}\left(x_{i}\right)\right]$ is $\min (n+1, m)$ it follows that the dimensionality of solutions in $\lambda$ of the linear equations $\left(^{*}\right)$ will be $m-\min (n+1, m)=\max (m-(n+1), 0)$. Thus if $m \leqq n+1$, then the solution is unique and $\mu$ is an extreme point. If $m>n+1$, then since a solution with $\lambda_{i}>0$ exists, it follows if $\sum_{i=1}^{m} z_{i} h_{j}\left(x_{i}\right)=0$ that $\lambda_{i} \pm \epsilon \boldsymbol{z}_{i}$ $\geqq 0$ and $\sum\left(\lambda_{i} \pm \epsilon z_{i}\right) h_{j}\left(x_{i}\right)=a_{j}$ for $\epsilon$ sufficiently small and hence $\lambda_{i}$ $=\left(\lambda_{i}+\epsilon z_{i}\right) / 2+\left(\lambda_{i}-\epsilon z_{i}\right) / 2$. This shows that the extreme points are given by precisely those measures of $L$ which are composed of not more than $n+1$ pure atomic measures. We have thus established:

THEOREM 5. The extreme points of the set $L$ of all positive regular measures over a topological space $X$ satisfying $\int d \mu(x)=1, \int h_{i}(x) d \mu=a_{i}$, $i=1, \cdots, n$, with $h_{i}(x)$ continuous, consist of those measures in $L$ with not more than $n+1$ points of increase provided that the rank of $\left\{h_{i}\left(x_{j}\right)\right\}$ is $n+1$ where $i, j=0,1, \cdots, n, h_{0}(x) \equiv 1$, and $x_{j}$ are distinct points of $X$.

\section{REFERENCES}

1. D. Blackwell, The range of a vector measure, Proceedings of the American Mathematical Society vol. 2 (1951).

2. A. Dvoretsky, A. Wald, and J. Wolfowitz, Relations among certain ranges of vector measures, Pacific Journal of Mathematics (1951).

3. L. Alaoglu, Weak topologies of normed linear spaces, Ann. of Math. (1940).

4. A. Liapounov, Sur les fonctions-vecteurs complement additives, Bull. Acad. Sci. USSR. vol. 4 (1940).

California Institute of Technology 